\title{
MicroRNA-34b functions as a tumor suppressor and acts as a nodal point in the feedback loop with Met
}

\author{
LI-GUANG WANG $^{1 *}$, YANG NI $^{1 *}$, BEN-HUA SU ${ }^{2}$, XUE-RU MU ${ }^{3}$, HONG-CHANG SHEN ${ }^{3}$ and JIA-JUN DU ${ }^{1,3}$ \\ Departments of ${ }^{1}$ Thoracic Surgery and ${ }^{2}$ Medical Engineering, ${ }^{3}$ Institute for Cancer Research, \\ Provincial Hospital Affiliated to Shandong University, Shandong University, Jinan, P.R. China
}

Received October 26, 2012; Accepted December 14, 2012

DOI: 10.3892/ijo.2013.1767

\begin{abstract}
MicroRNAs (miRNAs), as a class of naturally occurring small non-coding RNAs, play profound and pervasive roles in cancer initiation and progression. Extensive decrease in miRNA levels are frequently observed in human cancers, indicating that miRNAs may function intrinsically in tumor suppression. However, the underlying mechanisms of miRNA interactions with cellular pathways are still unclear. The expression of miR-34b in non-small cell lung cancer (NSCLC) tissues was detected using quantitative real-time PCR. The relations between miR-34b expression levels and pathological stage or lymph node metastasis were assessed using the Spearman correlation test. For in vitro studies, lung cancer cells were transfected with double stranded synthetic miRNA mimics (syn-hsa-miR-34b miScript miRNA) and scrambled controls. Immunohistochemistry was used to validate the related downstream proteins of miR-34b. The expression of miR-34b was lower in NSCLC tissues compared to that in pericarcinous tissues of lung cancer. Additionally, the Spearman correlation test showed that lower miR-34b expression was correlated with higher lymph node metastasis. In vitro gain-of-function experiments indicated that miR-34b suppressed cell proliferation by inducing cell apoptosis. IHC results showed association between lower miR-34b and overexpression of phospho-Met, p53 (phospho S392) and Mdm2. Consistent with the opposing correlation between the expression of miR-34b and lymph node metastasis in NSCLC, miR-34b may play an important role in NSCLC progression. Furthermore, miR-34b downregulates Met, with subsequent changes of downstream p53 (phospho S392) and Mdm2, and inversely p53 upregulates miR-34b in a feedback loop, which provides new insights into the roles of
\end{abstract}

Correspondence to: Dr Jia-Jun Du, Department of Thoracic Surgery, Institute for Cancer Research, Provincial Hospital Affiliated to Shandong University, Shandong University, 51 Weiliu Road, Jinan, 250021, P.R. China

E-mail: dujiajun@sdu.edu.cn

${ }^{*}$ Contributed equally

Key words: miR-34b, Met, p53, feedback loop, non-small cell lung cancer
miR-34 family members in the regulation of signaling pathways of NSCLC.

\section{Introduction}

miRNAs are a class of endogenous small non-coding RNAs (approximately 21-25 nucleotides in length), which are derived from longer transcripts termed pri-miRNAs and pre-miRNAs (1-5). miRNAs combine target mRNAs through partial complementarity to specific sequences located in the 3 ' untranslated region (3'-UTR) and act as post-transcriptional regulators of gene expression (6-9). It has been shown that human miRNA genes regions along with perturbed miRNA expression patterns have been detected in many human benign and malignant cancers (10). Therefore, it is of potential importance to elucidate the biological functions of miRNAs.

Additionally, miRNAs have been shown to play important roles in invasion and metastasis of cancer (11-15). For example, miR-155 may take part in the TGF- $\beta$-induced epithelial-mesenchymal transition (EMT) and in cell migration and invasion through targeting of the RhoA transcript (16). MiR-21 has the ability to stimulate cell invasion and metastasis in several tumor models, including breast cancer (12), colon cancer (17), and glioma (18). The pro-metastatic transcription factor TWIST1 is able to activate miR-10b, which is essential for TWIST1-induced EMT involved in promotion of cell motility and invasiveness (19). Tumor invasion and metastasis are the critical indicators that define the prognosis of cancer patients. Therefore, it is very important to understand the specific roles of miRNAs in cancer progression, and it could lead to the identification of predictive markers and the development of novel therapeutic strategies for patients with metastases.

In mammalians, the miR-34 family consists of three miRNAs which are encoded by two different genes: miR-34a is encoded by its own transcript, whereas miR-34b/c share the common primary one. In mice, miR-34a is highly expressed in brain tissues (20), whereas miR-34b/c is mainly expressed in lung tissues (21). These analyses also pointed out that miR-34a is expressed at higher levels than miR-34b/c, with the exception of the lung. miR-34 genes may be the important targets for other signaling pathways involved in normal life progression, however this remains to be determined by genetic analysis.

The aim of this study is to determine the role of miR-34b in the non-small cell lung cancer. In our study, the expres- 
sion level of miR-34b was significantly decreased in NSCLC tissues in comparison with pericarcinous tissues of lung cancer. In vitro gain-of-function experiments indicated that miR-34b functioned as a tumor suppressor and inhibited cell proliferations by inducing cell apoptosis. Thus, we analyzed the relations between miR-34b and Met and relevant proteins using IHC technology. The results suggest a miR-34b/Met axis with potential therapeutic implications.

\section{Materials and methods}

Samples. Twenty-nine pairs of NSCLC specimens and corresponding pericarcinous tissues of lung cancer (PTLC) were collected at the time of surgery and prior to chemotherapy. Specimens were obtained from patients in Shandong Provincial Hospital Affiliated to Shandong University from 2010 to 2011 with informed consent. All tissue samples were from untreated patients undergoing surgery and all clinicopathological information was available. The study was approved by the Hospitals Ethics Review Committee. Part of the tissue specimens were snap frozen in liquid nitrogen and stored at $-80^{\circ} \mathrm{C}$, and the remaining were fixed with formalin and embedded in paraffin.

Cell culture. Human NSCLC cell lines (A549 and SPC-A-1) were provided by Cell Bank for Chinese Academy of Sciences. A549 was grown in DMEM (Hyclone) and SPC-A-1 in 1640 (Hyclone) supplemented with $10 \%$ fetal bovine serum (Gibco) and 100 units $/ \mathrm{ml}$ penicillin and $1 \%$ streptomycin (Invitrogen) and maintained at $37^{\circ} \mathrm{C}$ with $5 \% \mathrm{CO}_{2}$.

Quantitative real-time polymerase chain reaction ( $q R T-P C R)$. Total RNA was obtained from NSCLC frozen tumor tissues, pericarcinous tissues of lung cancer and cell lines using TRIzol reagent (Invitrogen), according to the manufacturer's instructions. RNA concentration was determined spectrophotometrically, and integrity was checked by gel electrophoresis. RNA quality was confirmed in an Agilent 2100 Bioanalyzer (Agilent Technologies). The expression levels of the mature miRNAs were determined using TaqMan MicroRNA Assays (Applied Biosystems) and cDNAs were synthesized using the TaqMan miRNA RT kit based on the specific stem-loop RT primer design. Reverse transcriptase reactions contained $10 \mathrm{ng}$ RNA samples, $3 \mu \mathrm{l}$ stem-loop RT primers, $1.5 \mu \mathrm{l}$ of 10X RT buffer, $0.15 \mu \mathrm{l}$ of $100 \mathrm{mM}$ dNTPs, $1 \mu \mathrm{l}$ MultiScribe reverse transcriptase, $0.19 \mu \mathrm{l}$ RNase inhibitor and $4.16 \mu 1$ nuclease free water. The $15 \mu \mathrm{l}$ reactions were incubated for $30 \mathrm{~min}$ at $16^{\circ} \mathrm{C}, 30 \mathrm{~min}$ at $42^{\circ} \mathrm{C}, 5 \mathrm{~min}$ at $85^{\circ} \mathrm{C}$, and then held at $4^{\circ} \mathrm{C}$. The $20 \mu 1 \mathrm{PCR}$ reaction included $1.33 \mu \mathrm{l}$ RT product, $1 \mathrm{X}$ TaqMan Universal PCR master mix and $1 \mu 1$ primers and probe mix of the TaqMan MicroRNA Assay kit. The initial PCR step was a $10 \mathrm{~min}$ hold at $95^{\circ} \mathrm{C}$; then 40 cycles consisted of a 15 -sec denaturation step at $95^{\circ} \mathrm{C}$ followed by 1-min of annealing/ extension at $63^{\circ} \mathrm{C}$. The PCR reactions were run on a 7500 real-time PCR machine (Applied Biosystems) and analyzed using 7500 System SDS software. The miRNA expression level was normalized to the expression level of U6 small nuclear RNA (RNU6B). Primers used for hsa-miR-34b and RNU6B were purchased from Applied Biosystems. All reactions were performed in triplicate and included a negative control lacking cDNA.
Cell transfection. SPC-A-1 cell line was transfected with double stranded synthetic miRNA mimics (syn-hsa-miR-34b miScript miRNA) and scrambled controls (Qiagen) using HiPerFect transfection reagent (Qiagen) according to the manufacturer's protocol for overexpression. Approximately $5 \times 10^{5}$ cells were seeded and cultured in 6 -well plates. Complexes containing the mimics were added directly to each well at a final miRNA mimic concentration of $5 \mathrm{nM}$. Incubate the cells with the transfection complexes under their normal growth conditions. All groups were performed in triplicate.

Cell apoptosis. For cell apoptosis assay, SPC-A-1 cells were seeded in 6-well plates, both adherent and floating cells were harvested at $72 \mathrm{~h}$ after transfection, and stained with Annexin V-FITC (Clontech) and propidium iodide (PI) (Clontech) for $15 \mathrm{~min}$ in the dark at room temperature followed by flow cytometric analysis. The cell line experiment was performed at least three times depending on the reproducibility.

Immunohistochemistry (IHC). Antibodies against phosphoMet, p53 (phospho S392) and Mdm2 are rabbit polyclonal antibodies (Abcam). In brief, the slides were dewaxed, and endogenous peroxidase activity was then quenched with $3 \%$ $\mathrm{H}_{2} \mathrm{O}_{2}$. Tissue samples were heated in $1 \mathrm{mmol} / \mathrm{l}$ ethylenediaminetetraacetic acid (EDTA) buffer for $15 \mathrm{~min}$ in a water bath $\left(96-98^{\circ} \mathrm{C}\right)$ to retrieve antigens, and cross-reactivity was blocked with normal goat serum. The slides were then incubated overnight at $4^{\circ} \mathrm{C}$ with primary antibodies (1:500 for primary antibody). The subsequent steps were according to the instructions of Zymed (Streptavidin-Perosidase Method). The primary antibodies were replaced by normal serum or phosphate-buffered saline (PBS) as negative controls.

Evaluation of immunostaining. The criterion for a positive reaction was clear cytoplasm or/and nucleus staining. The samples with more than $10 \%$ of the tumor cells stained were considered to be positive.

Statistical analysis. A statistical analysis was done using the SPSS 18.0 statistical software package. For qRT-PCR data, the statistical analysis of miR-34b expression level in NSCLC tissues and PTLC tissues were $\log 2$ transformed. Values are expressed as the mean \pm SEM. Differences in miR-34b between NSCLC and PTLC were analyzed using two-sample t-test. Pearson $\chi^{2}$ and Fisher's exact tests were used to determine the correlation between miR-34b expression and clinical stage and lymph node metastasis status. Spearman correlation analysis was used to determine the correlation between miR-34b expression and levels of phospho-Met, pS392p53 and Mdm2. Other results were analyzed using independent sample t-test. $\mathrm{P}<0.05$ was defined as being significant.

\section{Results}

Downregulation of miR-34b expression in NSCLC. Compared with PTLC, a significant downregulation of miR-34b expression in NSCLC was noted. Of 29 matched cancer and normal tissues, the expressions of miR-34b in 27 cancer tissues were decreased in comparison with the matched PTLC $(\mathrm{p}<0.001)$ 


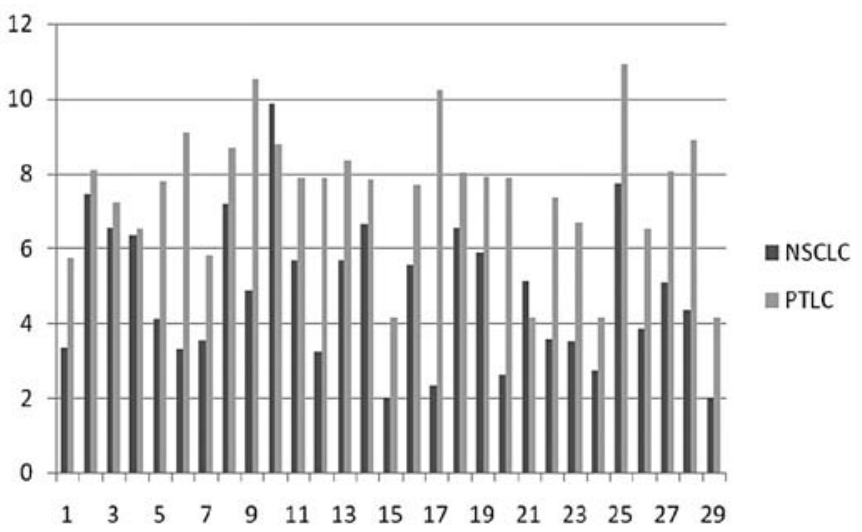

Figure. Downregulation of mature miRNA-34b in NSCLC tissue specimens. The comparison of miR-34b relative expressions between NSCLC tissues and matched PTLC epithelia in 29 patients is shown. The values represent the means of three replicates, and the relative expression values were calculated using the equation $R Q=2^{-\Delta \Delta C t} . P<0.05$.

(Fig. 1). The mean level of miR-34b in NSCLC was decreased about $66.11 \pm 4.31 \%$ compared to that in PTLC.

Correlations between miR-34b expression and clinicopathological characteristics of NSCLC. For further analysis, all patients were divided into 2 groups (miR-34b high and low expression) on the basis of the mean level of miR-34b expression in 29 NSCLC, and the clinicopathological characteristics of these groups were summarized. A significant association between the miR-34b expression level and lymph node metastasis was observed (Table I). In 8 cases without lymph node metastasis, $1(12.50 \%)$ has high level of miR-34b expression, whereas in 21 cases with lymph node metastasis, $12(57.14 \%)$ cases have high expression levels of miR-34b ( $\mathrm{P}=0.031)$. No correlation was observed between miR-34b expressions and gender, age, differentiation or pathologic TNM stage.

miR-34b transfection induces cell apoptosis in NSCLCs. Next, we examined the effects of ectopic miR-34b on the cell growth arrest by flow cytometry. Overexpression of miR-34b resulted in a significant increase in the proportion of FITC-annexin $\mathrm{V}$-positive cells and FITC-annexin V-negative/propidium iodide (PI)-positive cells, and a decrease in the proportion of FITC-annexin V-negative/PI-negative cells (Fig. 2), which indicated that overexpression of miR-34b may lead to apoptosis and necrosis. Taken together, the results suggest that miR-34b may inhibit proliferation of NSCLC cells mainly by inducing apoptosis.

phospho-Met, p53 (phospho S392) and Mdm2 are overexpressed corresponding to miR-34b reduction. Immunohistochemistry technology was used to examine the expression of phospho-Met, p53 (phosphoS392) and Mdm2 in clinical

Table I. Relations between miR-34b expression and clinicopathological characteristics in patients with NSCLC.

\begin{tabular}{|c|c|c|c|c|}
\hline \multirow[t]{2}{*}{ Characteristics } & \multirow[t]{2}{*}{ Patients } & \multicolumn{2}{|c|}{ miR-34b expression } & \multirow[t]{2}{*}{ P-value } \\
\hline & & High & Low & \\
\hline Gender & & & & 0.525 \\
\hline Male & 23 & 11 & 12 & \\
\hline Female & 6 & 2 & 4 & \\
\hline Age & & & & 0.897 \\
\hline$<60$ & 13 & 6 & 7 & \\
\hline$\geq 60$ & 16 & 7 & 9 & \\
\hline Differentiation & & & & 0.198 \\
\hline Well/ Moderate & 15 & 5 & 10 & \\
\hline Poor & 14 & 8 & 6 & \\
\hline Invasion depth & & & & 0.957 \\
\hline $\mathrm{T} 1$ & 6 & 3 & 3 & \\
\hline $\mathrm{T} 2$ & 14 & 6 & 8 & \\
\hline $\mathrm{T} 3, \mathrm{~T} 4$ & 9 & 4 & 5 & \\
\hline Lymph node metastasis & & & & 0.031 \\
\hline Positive & 21 & 12 & 9 & \\
\hline Negative & 8 & 1 & 7 & \\
\hline TNM stage & & & & 0.384 \\
\hline I & 6 & 2 & 4 & \\
\hline II & 7 & 2 & 5 & \\
\hline III, IV & 16 & 9 & 7 & \\
\hline
\end{tabular}


A

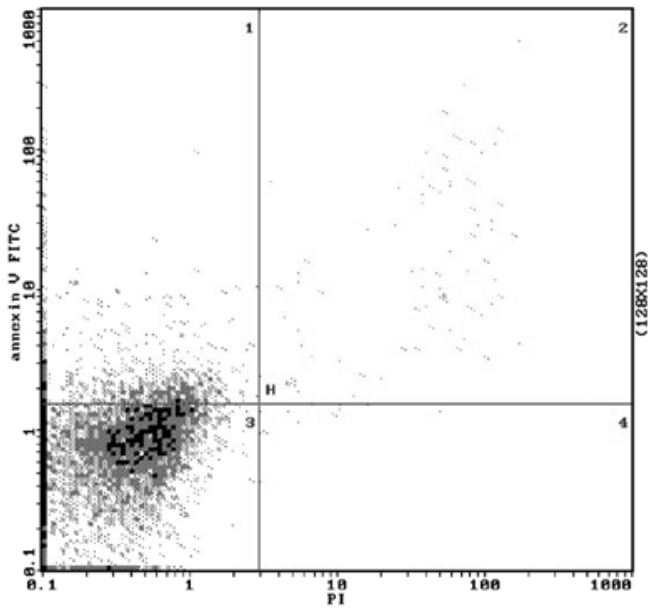

$\mathrm{C}$

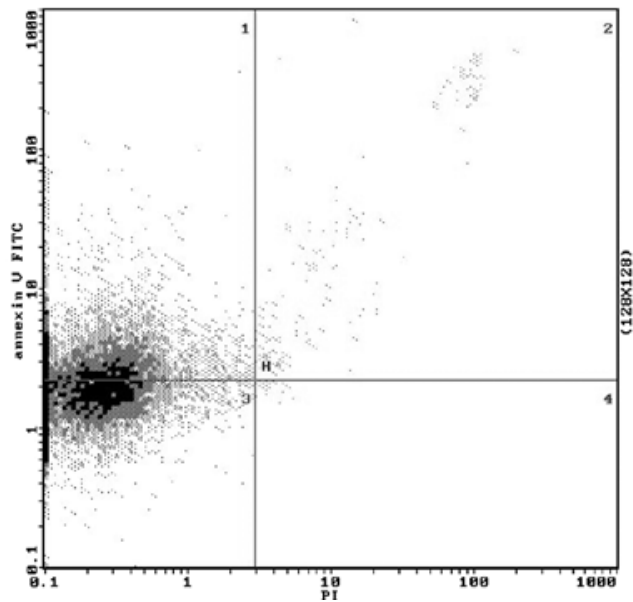

A

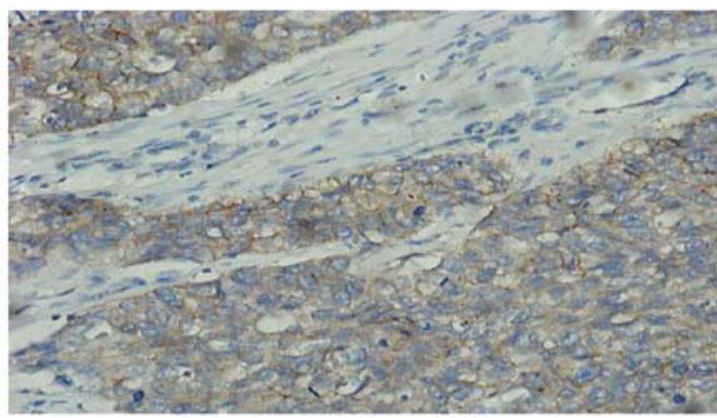

B

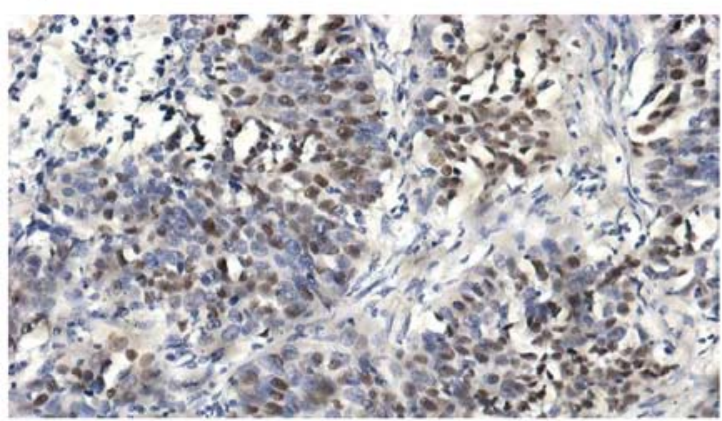

B

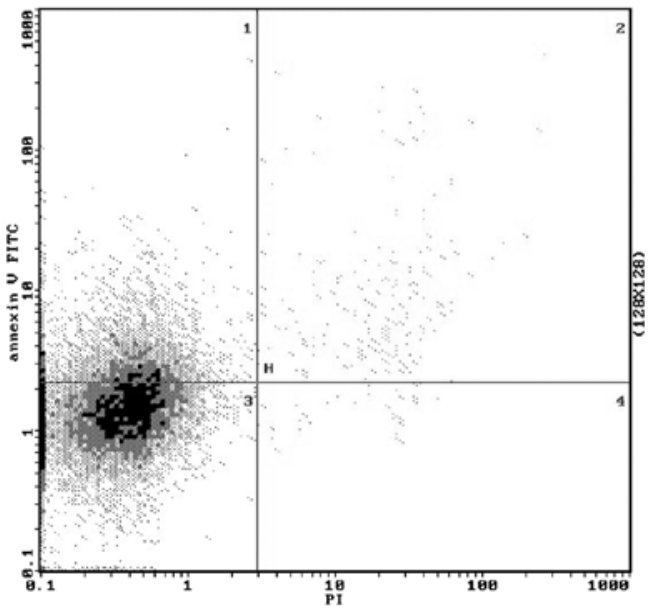

Figure 2. Effects of miR-34b on cell apoptosis. At $72 \mathrm{~h}$ post-transfection, cells were stained with FITC-conjugated annexin V and PI. Cell apoptosis was evaluated using flow cytometry. Cell death was more obvious in cells transfected with miR-34b than normal control miRNA groups and in cells transfected with only HiPerFect transfection reagent. (A) Normal control miRNA groups. (B) Cells transfected with only HiPerFect transfection reagent. (C) Cells transfected with miR-34b mimics and HiPerFect transfection reagent.

$\mathrm{C}$

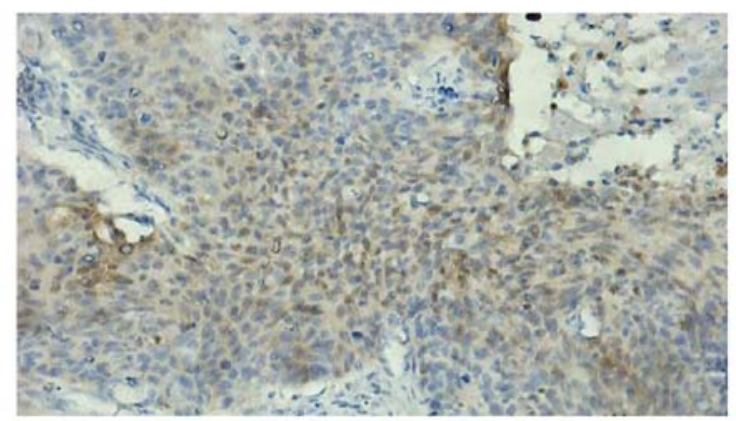

D

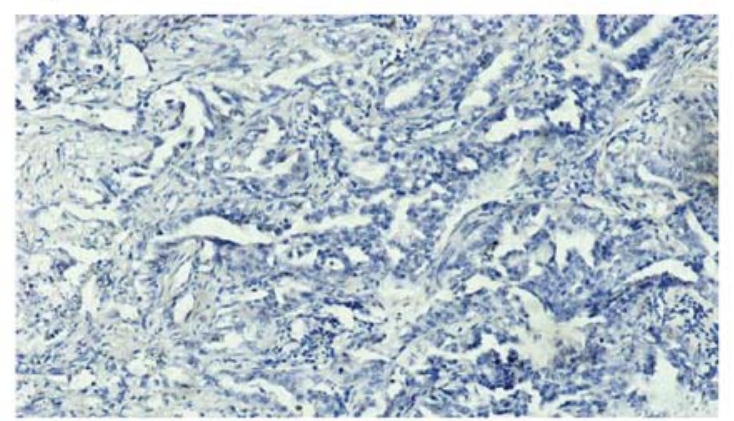

Figure 3. IHC staining of NSCLC using antibodies to phospho-Met, p53 (phosphor S392) and Mdm2. (A) Phospho-Met predominantly expressed in cell membrane and cytoplasm. (B) p53 (phosphor S392) positive staining in cell nucleus. (C) Mdm2 predominantly expressed in cytoplasm. (D) Negative control. 


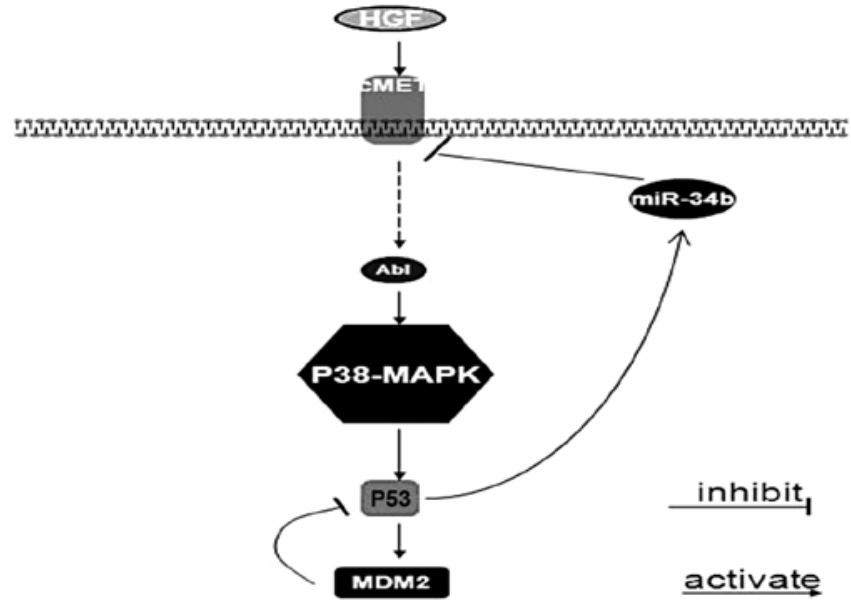

Figure 4. Integration of c-Abl-p38-p53 signaling in miR-34b/Met axis in NSCLC. miR-34b regulates the HGF-MET signal pathway, in which p53 could be modulated through phosphorylation of $\mathrm{p} 53$ on Ser392 by p38-MAPK and inversely p53 possesses anti-survival potential by upregulating miR-34b, subsequently miR-34b has an effect on Met in a feedback loop.

samples with NSCLC and matched PTLC (Fig. 3). There were positive expressions of phospho-Met in 44.8\% (13/29), pS392p53 in $58.62 \%$ (17/29) and Mdm2 in 79.31\% (23/29) of samples with NSCLC, respectively. In addition, Met IHC scoring was correlated with miR-34b level in samples with NSCLC $(\mathrm{P}=0.012)$. Spearman correlation analysis was used to determine the correlations between miR-34b expression and levels of phospho-Met, pS392p53 and Mdm2.

\section{Discussion}

It has been provn that miRNAs to function as important regulators of gene post-transcriptional regulation. Accumulated studies have indicated that miRNAs act as tumor suppressors or oncogenes depending on whether their specific targets act as oncogenes or tumor suppressors, suggesting that the aberrant expressions of several miRNAs might contribute to human carcinogenesis $(12,22)$. Thus, understanding of the specific miRNAs involved in the process of tumor development would provide significant insights for the diagnosis and treatment of patients with tumors. Our data showed that miR-34b functioned as a tumor suppressor and was correlated with tumor progression.

Additionally, as expected, NSCLC cells transfected with miR-34b mimics induced apoptosis, indicating that miR-34b had drastic effects on cell survival. The ectopic expression of either miR-34a or miR-34b/c led to substantial apoptosis and growth arrest (23). Previous studies have proved that ectopic miR-34b caused a cell cycle arrest in the G1 phase $(21,24)$. As apoptosis and cell cycle arrest are common end points of p53 signal pathway activation, miR-34 genes could be the potent regulators of tumor suppression by p53 (23). The induction of miR-34 genes permits p53 to regulate the expression of a large number of proteins. Furthermore, targeting of p53-induced mRNAs by miR-34 may affect p53 preventing an uncontrolled response of p53 activation (25).
Receptor tyrosine kinase (RTK) signaling is a core pathway frequently altered in cancer. Over recent years, therapeutic approaches based on compounds selectively targeting oncogenic RTKs have been developed. As RTKs share several effectors that participate in the oncogenic process and in drug response, an alternative strategy would rely on the identification of drug-treatable nodal points required for RTK-triggered tumorigenesis.

The Met-RTK and its ligand HGF have essential functions during embryogenesis and regenerative processes by regulating cell development, scattering, migration, angiogenesis, survival, proliferation and differentiation (26). Met is a target of miR-34b and is regulated by miR-34b combining Met mRNAs through partial complementarity to sequences located in the 3'-UTR of Met. It has been shown that c-Abl activation by Met triggers p53 phosphorylation on Ser392, which elevates the transcriptional activities of p53 and drives the transcriptional upregulation of $\mathrm{Mdm} 2$ and protection from cell death (27). Furthermore, p53 enhances the expression of miR-34 genes, which in turn mediates downregulation and degradation of Met. However, there is no increased expression level of miR-34b corresponding to the overexpression of pS392 observed in the samples with NSCLC. We suspect that miR-34b may be modulated by other effectors in cellular signal pathways, or phosphrylation on S392 decrease the transcriptional activities of p53 in lung cancer tissues.

miR-34b regulates the HGF-MET signal pathway, in which p53 could be modulated through phosphorylation of p53 on Ser392 by p38-MAPK and inversely p53 possesses anti-survival potential by upregulating miR-34b, subsequently miR-34b has an effect on Met in a feedback loop (Fig. 4). Collectively, Met and miR-34b work as key nodal points in p53 network, which provides new insights into therapeutic strategies of HGF-MET signal pathway and functional restoration of miR-34b is an effective novel therapy.

\section{Acknowledgements}

This study was supported by grants from the National Natural Science Foundation of China (81141100), Natural Science Foundation of Shandong Province of China (ZR2010HM067 and ZR2011HM077) and Provincial Science and Technology Foundation of Shandong (2011GGH21819).

\section{References}

1. Lee RC, Feinbaum RL and Ambros V: The C. elegans heterochronic gene lin-4 encodes small RNAs with antisense complementarity to lin-14. Cell 75: 843-854, 1993.

2. Lee Y, Ahn C, Han J, et al: The nuclear RNase III Drosha initiates microRNA processing. Nature 425: 415-419, 2003.

3. Kong W, Zhao JJ, He L and Cheng JQ: Strategies for profiling microRNA expression. J Cell Physiol 218: 22-25, 2009.

4. Hutvágner G, McLachlan J, Pasquinelli AE, Bálint E, Tuschl T and Zamore PD: A cellular function for the RNA-interference enzyme Dicer in the maturation of the let-7 small temporal RNA. Science 293: 834-838, 2001.

5. Grishok A, Pasquinelli AE, Conte D, et al: Genes and mechanisms related to RNA interference regulate expression of the small temporal RNAs that control C. elegans developmental timing. Cell 106: 23-34, 2001.

6. Schwarz DS, Hutvágner G, Du T, Xu Z, Aronin N and Zamore PD: Asymmetry in the assembly of the RNAi enzyme complex. Cell 115: 199-208, 2003. 
7. Ørom UA, Nielsen FC and Lund AH: MicroRNA-10a binds the 5'UTR of ribosomal protein mRNAs and enhances their translation. Mol Cell 30: 460-471, 2008.

8. Brennecke J, Stark A, Russell RB and Cohen SM: Principles of microRNA-target recognition. PLoS Biol 3: E85, 2005.

9. Bartel DP: MicroRNAs genomics, biogenesis, mechanism, and function. Cell 116: 281-297, 2004.

10. Calin GA, Sevignani C, Dumitru CD, et al: Human microRNA genes are frequently located at fragile sites and genomic regions involved in cancers. Proc Natl Acad Sci USA 101: 2999-3004, 2004.

11. Crawford M, Brawner E, Batte K, et al: MicroRNA-126 inhibits invasion in nonsmall cell lung carcinoma cell lines. Biochem Biophys Res Commun 373: 607-612, 2008.

12. Zhu S, Wu H, Wu F, Nie D, Sheng S and Mo YY: MicroRNA-21 targets tumor suppressor genes in invasion and metastasis. Cell Res 18: 350-359, 2008.

13. Tavazoie SF, Alarcón C, Oskarsson T, et al: Endogenous human microRNAs that suppress breast cancer metastasis. Nature 451: 147-152, 2008.

14. Nicoloso MS, Spizzo R, Shimizu M, Rossi S and Calin GA: MicroRNAs - the micro steering wheel of tumour metastases. Nat Rev Cancer 9: 293-302, 2009.

15. Valastyan S, Reinhardt F, Benaich N, et al: A pleiotropically acting microRNA miR-31, inhibits breast cancer metastasis. Cel 137: 1032-1046, 2009.

16. Kong W, Yang H, He L, et al: MicroRNA-155 is regulated by the transforming growth factor beta/ Smad pathway and contributes to epithelial cell plasticity by targeting RhoA. Mol Cell Biol 28 : 6773-6784, 2008.

17. Asangani IA, Rasheed SA, Nikolova DA, et al: MicroRNA-21 (miR-21) posttranscriptionally downregulates tumor suppressor Pdcd 4 and stimulates invasion, intravasation and metastasis in colorectal cancer. Oncogene 27: 2128-2136, 2008.
18. Gabriely G, Wurdinger T, Kesari S, et al: MicroRNA 21 promotes glioma invasion by targeting matrix metalloproteinase regulators. Mol Cell Biol 28: 5369-5380, 2008.

19. Yang J, Mani SA and Weinberg RA: Exploring a new twist on tumor metastasis. Cancer Res 66: 4549-4552, 2006.

20. Lodygin D, Tarasov V, Epanchintsev A, et al: Inactivation of miR-34a by aberrant CpG methylation in multiple types of cancer. Cell Cycle 7: 2591-2600, 2008.

21. Bommer GT, Gerin I, Feng Y, et al: p53-mediated activation of miRNA34 candidate tumor-suppressor genes. Curr Biol 17: 1298-1307, 2007

22. Zhang B, Pan X, Cobb GP and Anderson TA: microRNAs as oncogenes and tumor suppressors. Dev Biol 302: 1-12, 2007.

23. He L, He X, Lim LP, et al: A microRNA component of the p53 tumour suppressor network. Nature 447: 1130-1134, 2007.

24. Tarasov V, Jung P, Verdoodt B, et al: Differential regulation of microRNAs by 553 revealed by massively parallel sequencing: miR-34a is a p53 target that induces apoptosis and G1-arrest. Cell Cycle 6: 1586-1593, 2007.

25. Cohen SM, Brennecke J and Stark A: Denoising feedback loops by thresholding-a new role for microRNAs. Genes Dev 20: 2769-2772, 2006 .

26. Naran S, Zhang $X$ and Hughes $S$ J: Inhibition of HGF/MET as therapy for malignancy. Expert Opin Ther Targets 13: 569-581, 2009.

27. Furlan A, Stagni V, Hussain A, et al: Abl interconnects oncogenic Met and p53 core pathways in cancer cells. Cell Death Differ 18: $1608-1616,2011$. 